\title{
Measurement of Collision Conditions in Magnetic Pulse
}

\section{Welding Processes}

Joerg Bellmann ${ }^{1,3}$, Eckhard Beyer ${ }^{1,3}$, Joern Lueg-Althoff ${ }^{2}$, Soeren Gies ${ }^{2}$, A. Erman Tekkaya ${ }^{2}$ and Sebastian Schulze ${ }^{3}$

1. Institute of Manufacturing Science and Engineering, Technical University Dresden, Dresden 01062, Germany

2. Institute of Forming Technology and Lightweight Components, TU Dortmund University, Dortmund 44227, Germany

3. Fraunhofer IWS Dresden, Dresden 01277, Germany

\begin{abstract}
MPW (magnetic pulse welding) is a solid state joining technology that allows for the generation of strong metallic bonds, even between dissimilar metals. Due to the absence of external heat, critical intermetallic phases can largely be avoided. In this process, Lorentz forces are utilized for the rapid acceleration of at least one of the two metallic joining partners leading to the controlled high velocity impact between them. The measurement of the collision conditions and their targeted manipulation are the key factors of a successful process development. Optical measuring techniques are preferred, since they are not influenced by the prevalent strong magnetic field in the vicinity of the working coil. In this paper, the characteristic high velocity impact flash during MPW was monitored and evaluated using phototransistors in order to measure the time of the impact. The results are in good accordance with the established PDV (photon Doppler velocimetry) and show a good repeatability. Furthermore, the collision front velocity was investigated using adapted part geometries within a series of tests. This velocity component is one of the key parameters in MPW; its value decreases along the weld zone. With the help of this newly introduced measurement tool, the magnetic pressure distribution or the joining geometry can be adjusted more effectively.
\end{abstract}

Key words: Magnetic pulse welding, impact welding, process monitoring, collision conditions, dissimilar metal joining.

\section{Introduction}

The material mixture in modern lightweight structures requires adequate joining technologies. In case of dissimilar metals, conventional fusion welding processes are often difficult to apply due to differences in the melting point or the formation of intermetallic phases. These are unfavorable because of their brittleness but can largely be avoided using solid state joining technologies with no external heat input. For example, the high speed collision of two metallic parts can be utilized to create a "cold weld" with high strength. The generation of sound welds requires proper kinetic conditions during the impact in order to initiate the "jetting"-effect [1]. The jet removes debris from the material's surfaces. Shortly afterwards, the

Corresponding author: Joerg Bellmann, M.Sc., scientific staff member, research field: magnetic pulse welding. joining partners are pressed together and finally, the weld is formed. The most relevant parameters for impact welding include the impact velocity $v_{\mathrm{i}}$ with its radial component $v_{\mathrm{i}, \mathrm{r}}$ and the axial collision front velocity $v_{\mathrm{c}}$ that are shown in Fig. 1 . Both can be correlated via the collision angle $\beta$ as follows:

$$
0.5 \sin 2 \beta=\mathrm{v}_{\mathrm{i}, \mathrm{r}} / \mathrm{v}_{\mathrm{c}}
$$

Many parameter studies were performed for different material combinations in order to identify those combinations of $v_{\mathrm{c}}$ and $\beta$ that guarantee a good weld quality. Therefore, explosives were used for the flyer acceleration within an EXW (explosive welding) process. Varying the detonation velocity and the initial standoff $g$ (see Fig. 1) between flyer and parent resulted in a change of $v_{\mathrm{c}}$ and $\beta$, respectively. Welding windows were developed that indicate the parameter combinations for which welding can be expected. 


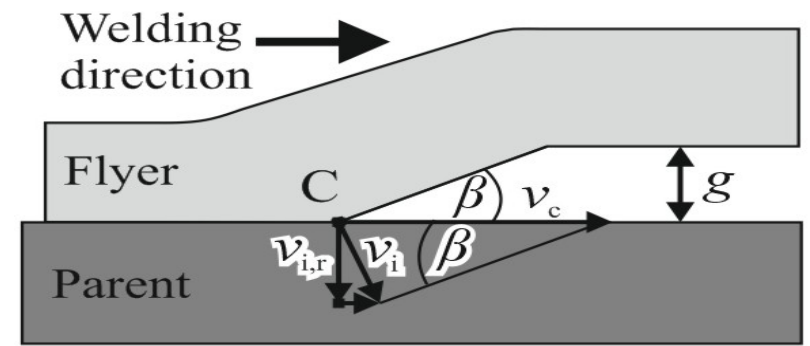

Fig. 1 Definition of collision parameters at the moving collision point $\mathrm{C}$.

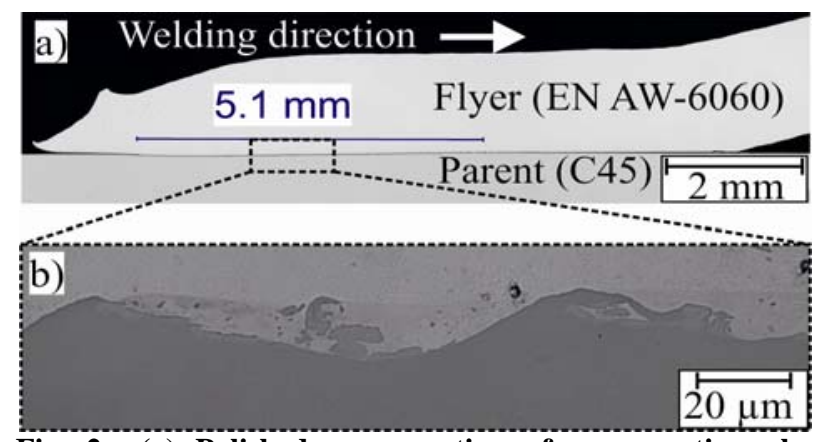

Fig. 2 (a) Polished cross section of a magnetic pulse welded area between aluminum (top) and steel (bottom); (b) High magnification shows a typical wavy interface.

Magnetic pulses can be used for the flyer acceleration as well, which is advantageous for small sized parts and industrial high volume production [2]. Fig. 2 shows the typical wavy interface between an aluminum flyer tube that impacted the steel parent rod during MPW (magnetic pulse welding) of a drive shaft dummy.

Transferring the favorable collision conditions from established EXW welding windows to the MPW process is quite challenging due to the multitude of influencing factors on the flyer forming process including mechanical properties, geometry as well as the time and place depended intensity of the magnetic field. In this context, coupled numerical simulations are a powerful tool for process modeling and can take electromagnetic, mechanical [3, 4], and even thermal interactions into account. Only when these models are validated by experimental results, they should be used for further studies.

There are already some principles available for direct and indirect measurement of the relevant velocity components at the point of impact [5]. For example, electro conductive foils on the parent surface [6] or contact pins [7] may act as switches when they get hit by the flyer and generate certain on-off-signals that can be used for an analysis of the process sequence. Those techniques are difficult to apply especially on small sized parts and for short welding zones. Furthermore, all electrical signals in the vicinity of the working coil are influenced by the strong magnetic field [8] and cables have to be shielded. Therefore, optical methods are preferred but need optical accessibility to the flyer surface. PDV (photon Doppler velocimetry), for example, is used for the measurement of surface velocities up to several kilometers per second with a very high temporal resolution [9]. The laser based recording of the radial impact velocity $v_{\mathrm{i}, \mathrm{r}}$ via PDV is an established method and can be done in two different ways. In the first setup the laser beam is guided directly [4] or via mirrors [10] through holes in the parent part towards the inner flyer surface. The second principle requires small boreholes for the collimators in the concentration zone of the working coil to ensure optical access to the outer flyer surface, see Fig. 3. Both principles show disadvantages either because they require a certain specimen size or due to increased current densities and a lower stiffness in the vicinity of the bore holes in the coils. Furthermore, it is not possible to measure $v_{\mathrm{c}}$ directly with a PDV system because of the oblique reflection of the laser beam.

To overcome these challenges, a measuring tool was developed that takes advantage of the HVI (high velocity impact) flash. This phenomenon naturally occurs during the collision of two metallic parts with a certain velocity due to the generation of a plasma [11] or activated particles within the jet [12]. Bellmann et al. [13] showed that the time dependent light intensity during MPW of aluminum and steel parts correlates with the radial impact velocity $v_{\mathrm{i}, \mathrm{r}}$ and the welding result. For a detailed understanding of the process conditions and their targeted manipulation it is also 
necessary to measure the collision front velocity $v_{\mathrm{c}}$. In a first step, small bore holes were implemented in the parent part to detect the on-off light signal while the flyer passed over each hole [5]. This approach was difficult to apply for small parts. Instead, a tool for the direct manipulation of the flash intensity and its analysis was developed. This was done with the help of sharp edges at the parent material and has a similar effect like a segmented flyer used by Appelgren et al. [14]. They evaluated the light intensity variation in a small helical magnetic flux-compression generator and calculated the axial contact point velocity that occurs while the expanding armature shortens the stator coil turn by turn. A direct transfer to MPW was not possible due to the different length scales, the shape of the flyer, and the absence of a rotating collision point over the circumference that generates the characteristic alternating light intensity in their setup. Thus, the present paper shows a modified method demonstrating the applicability of the HVI flash detection for collision velocity measurements within a series of experiments. Furthermore, the repeatability of the measuring device is evaluated and compared to the established PDV-method. Finally, modified experiments are described which correlate the flash development and the welding result to get more insight into the bonding mechanism of MPW.

\section{Experimental Setup}

\subsection{Materials and Machines}

Joining experiments were performed in order to correlate the characteristics of the HVI flash and the PDV signal. Therefore, aluminum flyer tubes were compressed towards steel cylinders with the setup depicted in Fig. 3. The chemical compositions of the alloys are given in Table 1 .

Both flyer and parent material were used in uncoated state and were cleaned in ethanol before the joining experiments to remove debris from the workpieces' surfaces. The geometric setup with an input energy of $10.2 \mathrm{~kJ}$ used by Bellmann et al. [17] lead to collision parameters within the welding window and served as a basis for the present investigations. The magnetic pressure was generated by a single turn working coil made of a $\mathrm{CuCrZr}$ alloy (2.1293) with an integrated PDV probe placed in the concentration zone as shown in Fig. 5.

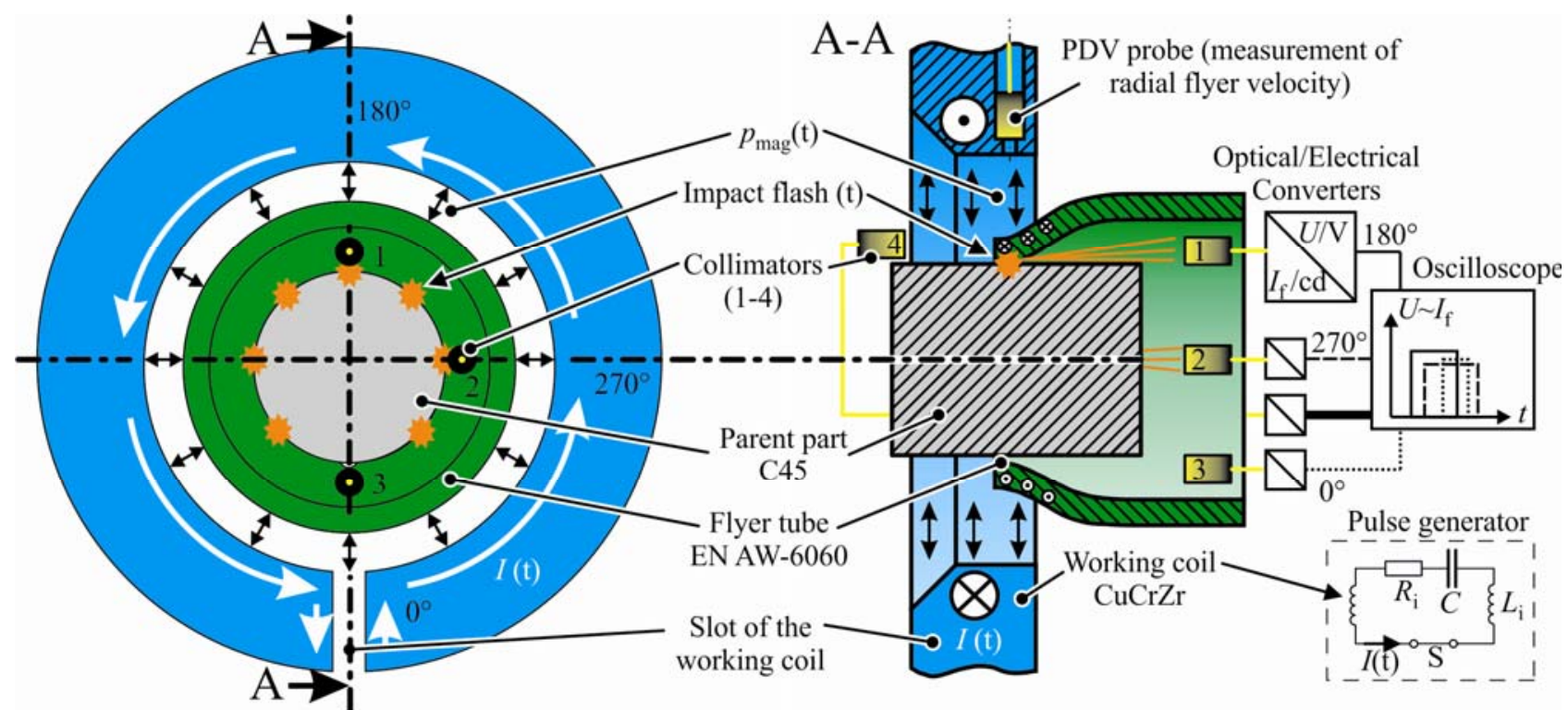

Fig. 3 Setup for MPW of tubes to cylinders with PDV probe for velocity measurement and collimators (1-4) for flash detection during and after the impact. 
Table 1 Aluminum EN AW-6060 alloy composition [15] and steel C45 (1.0503) alloy composition [16].

\begin{tabular}{llll}
\hline $\begin{array}{l}\text { Flyer part EN AW-6060, } \mathrm{T4}^{\mathrm{a}} \text {, quasi-static yield strength approx. } \\
60 \mathrm{MPa}^{\mathrm{b}}\end{array}$ & Parent part C45 (1.0503), normalized, surface polished $\left(\mathrm{R}_{\mathrm{a}}=1\right)$ \\
\hline Element & Weight \% & Element & Weight \% \\
\hline $\mathrm{Mg}$ & $0.35-0.6$ & $\mathrm{C}$ & $0.42-0.5$ \\
$\mathrm{Mn}$ & $\leq 0.1$ & $\mathrm{Mn}$ & $0.5-0.8$ \\
$\mathrm{Fe}$ & $0.1-0.3$ & $\mathrm{P}$ & $<0.045$ \\
$\mathrm{Si}$ & $\mathrm{S}$ & $<0.045$ \\
$\mathrm{Cu}$ & $\mathrm{Su}-0.6$ & $\mathrm{Si}$ & $<0.4$ \\
$\mathrm{Zn}$ & $\leq 0.1$ & $\mathrm{Ni}$ & $<0.4$ \\
$\mathrm{Cr}$ & $\leq 0.15$ & $\mathrm{Cr}$ & $<0.4$ \\
$\mathrm{Ti}$ & $\leq 0.05$ & $\mathrm{Mo}$ & $<0.1$ \\
\hline
\end{tabular}

(a) T66 heat treated: one hour at $500{ }^{\circ} \mathrm{C}$ and naturally aged; (b) determined by tube tensile test.

Table 2 Characteristic of pulse generator BMAX MPW 50/25.

\begin{tabular}{lll}
\hline Setup & Value & Unit \\
\hline Capacitance & 160 & $\mu \mathrm{F}$ \\
Resistance (short circuit) & 2 & $\mathrm{~m} \Omega$ \\
Output stage inductance (short circuit) & 58 & $\mathrm{nH}$ \\
Maximum charging energy & 32 & $\mathrm{~kJ}$ \\
Applied charging energy $-E$ & $4.5 \ldots 10.2$ & $\mathrm{~kJ}$ \\
Corresponding maximum tool coil current $-I_{\max }(E)$ & $\approx 35 \cdot E(\mathrm{~kJ})+218$ & $\mathrm{kA}$ \\
Discharge frequency with coil and workpieces $-f_{\text {discharge }}$ & 20 & $\mathrm{kHz}$ \\
\hline
\end{tabular}

The current was supplied by a pulse generator with its characteristic values listed in Table 2. Current measurements were conducted for each trial using a Rogowski current probe CWT 3000 B from Power Electronic Measurements Ltd. leading to an empirical correlation between the charging energy $E$ and the maximum tool coil current $I_{\max }$ for the investigated interval, as shown in Table 2.

The rising current signal triggered the recording of both the PDV signal and the light intensities. Therefore, the optical signals were collimated at the positions 1-4 and transmitted with four optical fibers (Polymer cladding fiber PCF, fiber diameter $0.2 \mathrm{~mm}$, length $7 \mathrm{~m}$, manufactured by ofs) to silicon phototransistors (SDP8405, Honeywell). Due to their photosensitivity, the transistors acted as optoelectrical converters and thus generated signals that can be correlated to the current signal from the tool coil, see Fig. 4. For each channel, the voltage was measured at a $220 \Omega$ resistor that was series connected with the phototransistor and a $9 \mathrm{~V}$ battery and thus reached maximum values of $9 \mathrm{~V}$. Three characteristic values were defined for each flash measurement: the flash appearance time $t_{\mathrm{f} \text {,start }}$, the flash duration $t_{\mathrm{f}}$ and the maximum intensity $I_{\mathrm{f}, \max }$. The end of the flash was defined to be at the steep decrease of the light intensity that was equivalent to approx. $4 \mathrm{~V}$ for all tests.

In a preliminary experiment according to Fig. 3 it was shown that no flash could be detected by collimator 4 , which is aligned to the left side of the free flyer edge. It can be concluded that the free flyer edge collided with the parent part first and a single collision front propagated to the right. This collision mode is called single front process [3] and is a prerequisite for the correct interpretation of the following experiments.

The aim of these investigations shown in the present paper is to analyze the axial collision point velocity $v_{\mathrm{c}}$ by HVI measurement. This requires modified parent geometries as shown in Fig. 5 in order to manipulate the light intensity. The increase of the distance $d$ between the flyer edge and the parent edge from 0 to $4 \mathrm{~mm}$ (see Fig. 5) postpones the time of impact and $t_{\mathrm{f} \text {,start }}$, respectively. Thus, the collision 
times of defined points at the inner surface of the flyer can be measured resulting in a time-position curve and $v_{\mathrm{c}}$-position curve. Furthermore, the step length $l$ on the parent is increased gradually from 0.5 to $8 \mathrm{~mm}$ to investigate the flash evaluation after the initial impact at $d=0 \mathrm{~mm}$.

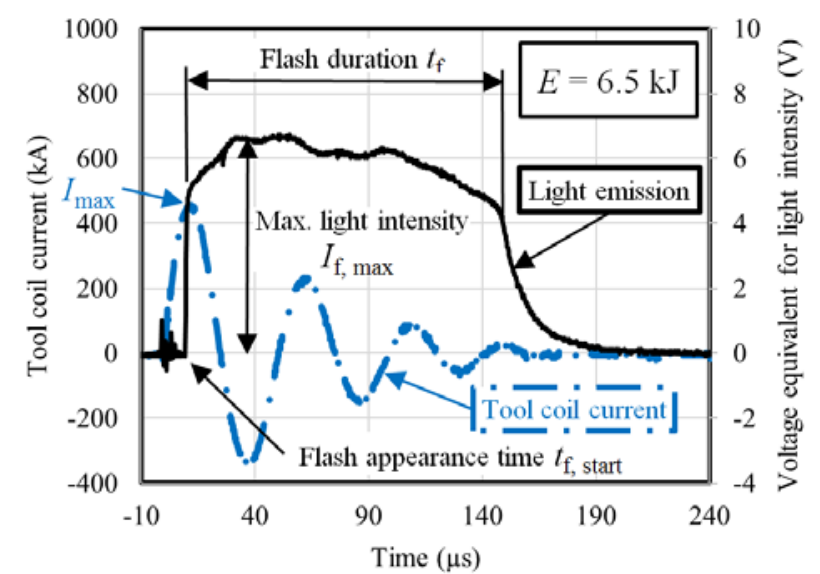

Fig. 4 Example of tool coil current and light intensity vs. time.

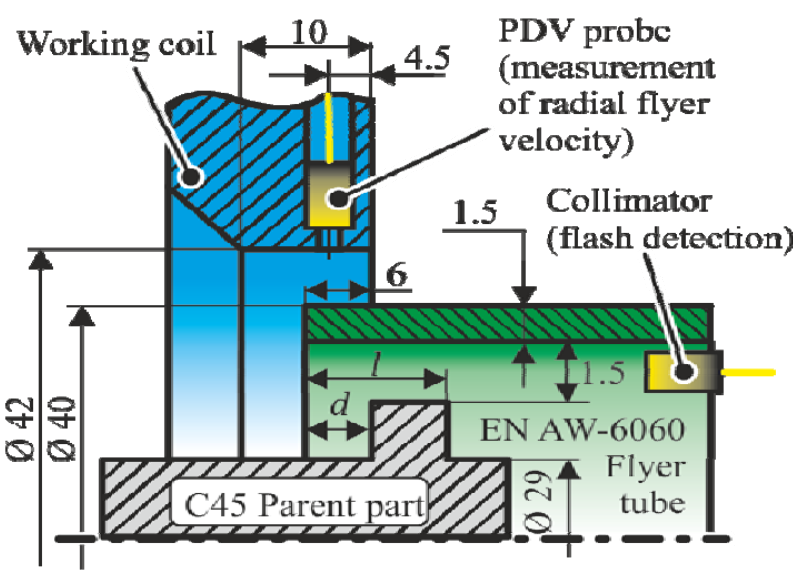

Fig. 5 Geometrical definitions of the modified welding setup and location of the PDV probe for measurement of the radial flyer velocity (not scaled, all values in $\mathbf{m m}$ ).

\subsection{Identification of Required Energy}

The required energy for a circumferential weld was investigated in preliminary tests. The results are listed in Table 3. As expected, an increased charging energy leads to an increased tool coil current, a higher flyer acceleration and an earlier collision. A charging energy of $6.5 \mathrm{~kJ}$ was identified to be sufficient for a circumferential weld with a width of $2 \mathrm{~mm}$ starting from $1 \mathrm{~mm}$ next to the free flyer edge. This energy level was chosen for the following experiments. It should be noted that the flash appeared later at the coils slot $\left(0^{\circ}\right)$ due to the reduced magnetic field and acceleration, respectively.

\subsection{Repeatability}

The introduction of the flash detection as a new measurement system for process monitoring of MPW requires a repeatability study and a validation with an established method like PDV. The results based on five repetitions are shown in Table 4.

Comparing the collision times recorded at $180^{\circ}$ reveals a slightly lower standard deviation for the flash detection method than the PDV measurement. The mean values differ about $0.5 \mu$ s due to the delay of the flash establishment and the optoelectrical conversion. Since this delay is systematical, it will have no influence on the determination of $v_{\mathrm{c}}$ in the following experiments. It can be concluded that the evaluation of $t_{\mathrm{f} \text {,start }}$ is a reliable tool for collision time measurement, although the flash duration and $I_{\mathrm{f}, \max }$ show larger deviations.

Table 3 Influence of the charging energy on the collision time, flash characteristic and welding result (according setup in Fig. 3).

\begin{tabular}{lllll}
\hline & Unit & Range & & \\
\hline Capacitor charging voltage $-U$ & $\mathrm{kV}$ & 7.5 & 9 & 11.3 \\
Capacitor charging energy $-E$ & $\mathrm{~kJ}$ & 4.5 & 6.5 & 10.2 \\
Maximum tool coil current $-I_{\max }$ & $\mathrm{kA}$ & 380 & 460 & 590 \\
$t_{\mathrm{f} \text {,start } 180^{\circ} \text { (Collimator 1) }}$ & $\mu \mathrm{s}$ & 11.6 & 10.7 & 9 \\
$t_{\mathrm{f} \text {,start }} 0^{\circ}$ (Collimator 2) & $\mu \mathrm{s}$ & 12.8 & 11.1 & 10 \\
Circumferential weld & & no & yes & yes \\
\hline
\end{tabular}


Table 4 Repeatability within five tests (according setup in Fig. 3).

\begin{tabular}{llll}
\hline & Mean & Standard deviation & Variance \\
\hline Capacitor charging voltage $-U$ & $9 \mathrm{kV}$ & $0 \mathrm{kV}$ & $0.0 \%$ \\
Capacitor charging energy $-E$ & $6.50 \mathrm{~kJ}$ & $0.00 \mathrm{~kJ}$ & $0.0 \%$ \\
Maximum tool coil current $-I_{\max }$ & $430 \mathrm{kA}$ & $4 \mathrm{kA}$ & $0.9 \%$ \\
Radial collision velocity (PDV-measurement) $-v_{\mathrm{i}, \mathrm{r}}$ & $395 \mathrm{~m} / \mathrm{s}$ & $15 \mathrm{~m} / \mathrm{s}$ & $3.8 \%$ \\
Collision time $\left(180^{\circ}\right.$ PDV) & $10.32 \mu \mathrm{s}$ & $0.26 \mu \mathrm{s}$ & $2.6 \%$ \\
$t_{\mathrm{f}, \mathrm{start}} 180^{\circ}($ Collimator 1$)$ & $10.80 \mu \mathrm{s}$ & $0.25 \mu \mathrm{s}$ & $2.3 \%$ \\
$t_{\mathrm{f}} 180^{\circ}($ Collimator 1$)$ & $183.20 \mu \mathrm{s}$ & $22.64 \mu \mathrm{s}$ & $12.4 \%$ \\
$I_{\mathrm{f}, \max } 180^{\circ}$ (Collimator 1$)$ & $7.01 \mathrm{~V}$ & $0.68 \mathrm{~V}$ & $9.7 \%$ \\
\hline
\end{tabular}

\subsection{Influence of the Parent Geometry}

The usability of the data generated with this new measurement principle is only ensured, if the applied contour of the parent part does not alter the forming behavior of the flyer compared to the original joining geometry. This might not completely be fulfilled since the free flyer edge moves on in these experiments and might affect the forming behavior of the remaining flyer segment. To study the influence of the modified parent contour, the collision times of defined flyer points with the original parent part (Fig. 3) were compared with the corresponding collision times of the flyer with the contoured parent edge (Fig. 5). 3D simulation models were prepared with the commercial software LS-DYNA (version R 8.1), which has the advantage of featuring an integrated electromagnetism module combining Finite and Boundary Element Methods [18]. The geometries of the coil, flyer and parent parts as well as the tool coil current curve recorded during the experiments $\left(I_{\max }=460 \mathrm{kA}\right)$ served as input. The original parent contour was compared with a series of contoured parent parts, where the edge length $d$ was gradually increased as shown in Fig. 6. It should be noted that welding was not considered in the simulation and consequently the flyer edge bounced back from the parent after the impact. This effect does not bother the comparison between the original and contoured parent part for $d<4 \mathrm{~mm}$, since the collision point reaches $d=4 \mathrm{~mm}$ before the flyer edge gets separated from the parent again.

The evaluation of the simulation results showed that the magnetic field intensities on the inner flyer surface at the free edge were reduced by the contour due to the enlarged volume of air. Consequently, the resulting magnetic pressure on the flyer outside was increased, leading to earlier collisions with the contoured parent parts as shown in Fig. 6 at five specific locations. The delay between the original and the contoured parent part is not constant but varies between 0.19 and $0.31 \mu \mathrm{s}$. Thus, a difference between the calculated mean front velocities appears with a maximum of $213 \mathrm{~m} / \mathrm{s}$ for $1 \mathrm{~mm}<d<2 \mathrm{~mm}$ which is equal to $9 \%$ variance. Nevertheless, the decrease of the collision front velocity from 3,000 to 1,500 respectively $1,300 \mathrm{~m} / \mathrm{s}$ within $4 \mathrm{~mm}$ is clearly visible in both cases. The corresponding approximation for the original parent contour with a quadratic equation has a coefficient of determination $\mathrm{R}^{2}=1.00$. Thus, this approach is likely to represent the collision front propagation within the investigated interval.

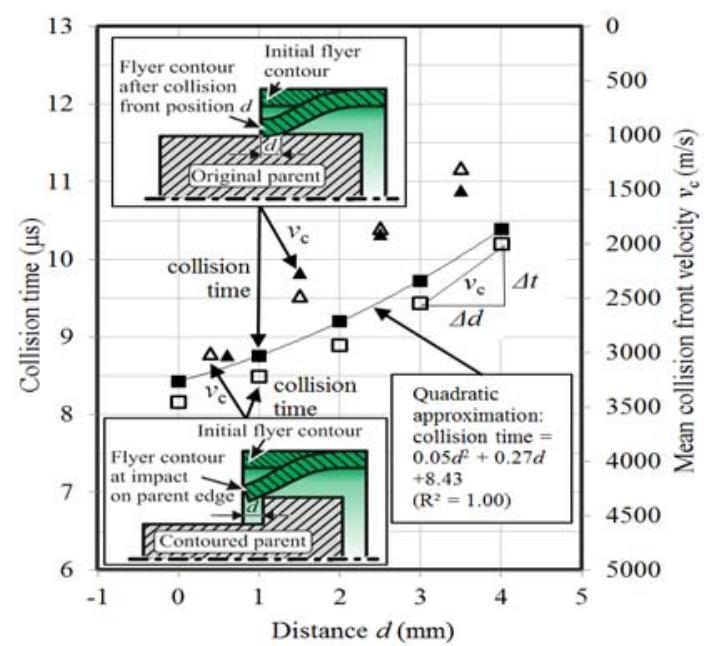

Fig. 6 Influence of the parent part's geometry on the simulated collision times and calculated mean collision front velocity $v_{\mathrm{c}}$. 


\section{Results and Discussion of the Flash Detection Experiments}

\subsection{Monitoring of the Axial Flyer Deformation}

The flash appearance times $t_{\mathrm{f}, \text { start }}$ at three positions of the working coil were evaluated for collision locations $d$ between 0 and $4 \mathrm{~mm}$. The results are plotted in Fig. 7.

Compared to the simulated collision times, the flash occurred 3.1 to $4.5 \mu$ s later, probably due to aging effects prior to the experiments resulting in differences between the real material's mechanical properties and the material model implemented in the simulation. But since these effects were present during the complete series of experiments, the evaluation of those experimental results is still valid. And the quadratic approximation has a coefficient of determination $\mathrm{R}^{2}=0.99$, which is slightly lower compared to the simulation results. One reason might be small variations in the tool coil current that are not considered in the simulations. The collision at the coil's slot $\left(0^{\circ}\right)$ shows a delay compared to the $180^{\circ}$ direction, probably due to the reduced magnetic pressure. The large difference for $d=2 \mathrm{~mm}$ might be a result of an off-centered part arrangement, but does not affect the course of the plotted mean value of $t_{\mathrm{f} \text {,start }}$. Thus, the mean values of the experiments can be used for the calculation of $v_{\mathrm{c}}$.

The calculated mean collision front velocities from the experiments and simulation are plotted in Fig. 8, combined with the location of the welded area. During the experiments, $v_{\mathrm{c}}$ decreased significantly from 2,300 to $1,400 \mathrm{~m} / \mathrm{s}$ within the first $3 \mathrm{~mm}$ and ended up at $600 \mathrm{~m} / \mathrm{s}$ for $3 \mathrm{~mm}<d<4 \mathrm{~mm}$. The collision front velocities deduced from simulations are partly up to $900 \mathrm{~m} / \mathrm{s}$ higher. One reason might be the material model that was not exactly fitted to the real material's properties as already explained before, and the neglect of the actual welding effect. Nevertheless, Fig. 8 suggests a lower boundary for a successful weld in the given configuration between 600 and $1,400 \mathrm{~m} / \mathrm{s}$ or
1,500 and $1,900 \mathrm{~m} / \mathrm{s}$ for the experiment and simulation, respectively. Probably, a finer graduation of $d$ during the experiments could localize the lower boundary more precisely. However, an accurate correlation between the end of the welding process and $v_{\mathrm{c}}$ is still challenging since the end of the welded zone is not always clearly defined. Furthermore, $v_{\mathrm{c}}$ is not the only relevant parameter for MPW. The radial impact velocity $v_{\mathrm{i}, \mathrm{r}}$ and $\beta$ have to be taken into account as well. Another interesting effect took place at the initial impact zone for $0 \mathrm{~mm}<d<1 \mathrm{~mm}$. In this area, no welding was observed, although the collision front velocity is above the lower limit and below the upper welding boundary of $5,100 \mathrm{~m} / \mathrm{s}$, defined by the speed of sound of the involved materials. These finding is in good correlation with literature [19] and will be explained by the observations in the next chapter.

The developed measuring principle offers an efficient method to check whether the deformation and collision behavior of the flyer allows for a weld establishment or not. Based on that, the effect of certain tools for the targeted manipulation of the flyer movement, e.g. adapted magnetic pressure profiles or surface contours, can be evaluated.

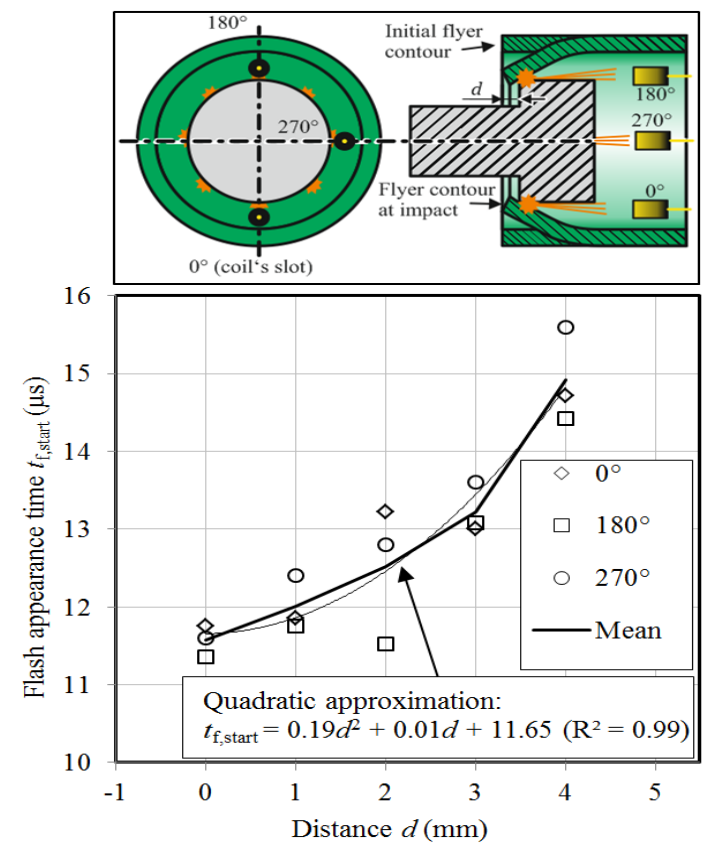

Fig. 7 Influence of the initial collision location $d$ on the flash appearance time $t_{\mathrm{f}, \text { start }}$. 


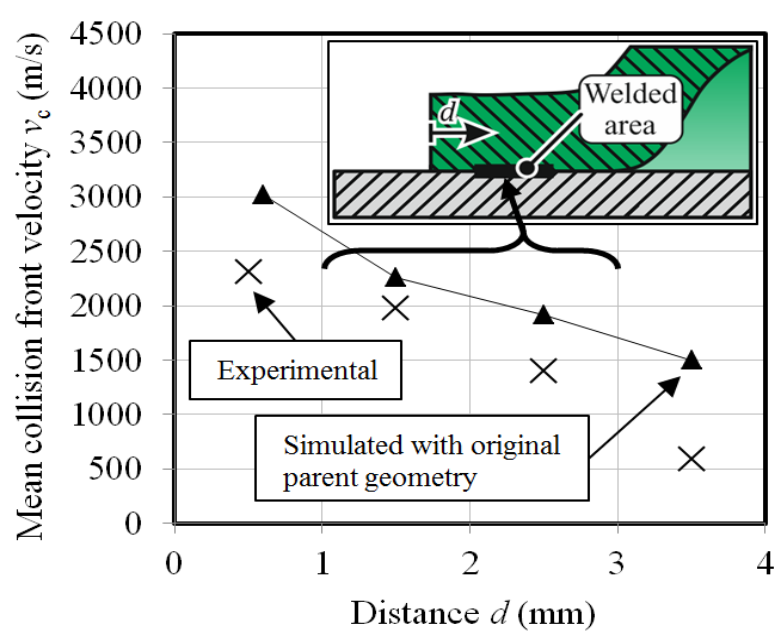

Fig. 8 Comparison of mean collision front velocities based on simulated and experimental collision times.

\subsection{Development of the Flash}

A second series of experiments was conducted with a gradually increasing collision length $l$ varying from 0.5 to $8 \mathrm{~mm}$. Then, the courses of the flashes (depicted in Fig. 9) were evaluated. During the experiment with $l=0.5 \mathrm{~mm}$, the flyer probably did not hit the intended step but just the inner contour of the parent part since the flash occurred not as intended at $11 \mu \mathrm{s}$ but at $17 \mu \mathrm{s}$. For $l=1.0 \mathrm{~mm}$, the flyer probably touched the parent edge and initiated a weak flash followed by the second impact and a significant rise of the light intensity. Increasing the collision length $l$ to $1.5 \mathrm{~mm}$ caused an immediate flash appearance. Since no significant changes of $I_{\mathrm{f}, \max }$ or $t_{\mathrm{f}}$ appeared above $l=3 \mathrm{~mm}$, this light intensity curve is representative for $l=3$ to $8 \mathrm{~mm}$ and is in good correlation with the joining experiments of the original parent contour.

The results indicate that the flash is visibly intensified between $l=1 \mathrm{~mm}$ and $l=1.5 \mathrm{~mm}$, probably because more particles are activated during the movement of the collision front and form the light emitting. These findings might also explain the delay of the weld formation with $1 \mathrm{~mm}$. The jet or flash, respectively, is too weak for a sufficient surface activation in the immediate vicinity of the initial impact.

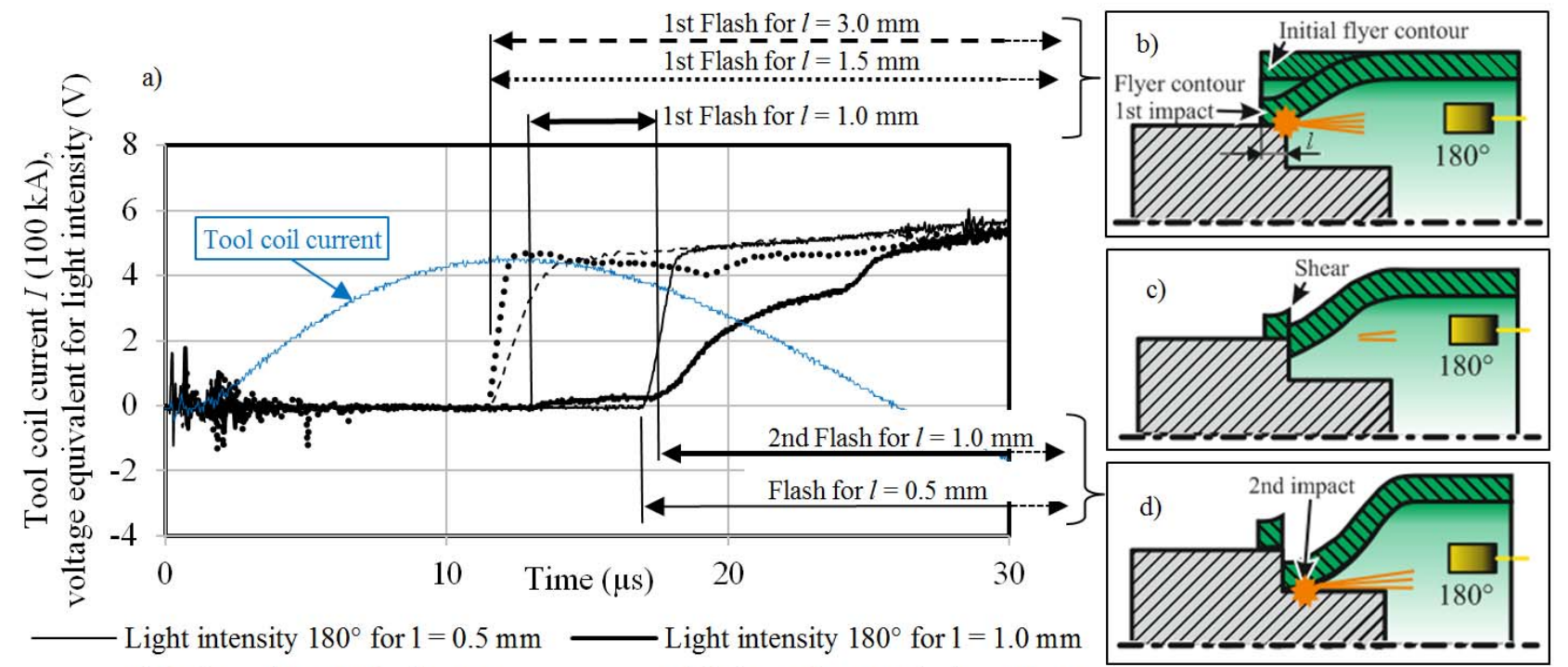

...... Light intensity $180^{\circ}$ for $1=1.5 \mathrm{~mm}-\cdots$ Light intensity $180^{\circ}$ for $1=3.0 \mathrm{~mm}$

Fig. 9 (a) Comparison of the flash intensities for collision lengths $I$ of $0.5 \mathrm{~mm}, 1.0 \mathrm{~mm}, 1.5 \mathrm{~mm}$ and $3.0 \mathrm{~mm}$; (b) Flyer contour at first impact; (c) Shearing of the flyer at diminished flash; (d) Flyer contour at second impact. 


\section{Conclusion and Outlook}

The radial impact velocity $v_{\mathrm{i}, \mathrm{r}}$ and the collision front velocity $v_{\mathrm{c}}$ have to be adjusted accurately at the MPW process in order to generate a sound weld. PDV systems are established tools for the measurement of the radial impact velocity, whereas a direct application for measuring $v_{c}$ is not possible. Conductive foils that are shorted when the flyer hits the parent part are difficult to apply and to shield against the strong electromagnetic field.

A measuring tool was developed that overcomes these issues and takes advantage of the high velocity impact flash. In preliminary tests, a good correlation of the flash appearance time $t_{\mathrm{f} \text {,start }}$ with the collision time detected by the PDV system was demonstrated, while the standard deviation was even below the PDV results after a series of five tests. The new method is easy to apply since no modifications of the coils are required. Thus, unwanted increases in the current densities or a lowered stiffness in the concentration zone can be avoided and measurements at the coil's slot can be performed as well.

The flash detection tool was used for adapted parent parts in order to investigate the flyer forming process on the parent surface that leads to the collision front velocity $v_{\mathrm{c}}$. Simulations showed that the adaption of the parent part distorts $v_{\mathrm{c}}$ up to $210 \mathrm{~m} / \mathrm{s}$ compared to the original parent geometry. This error is acceptable since it does not invalidate the finding that $v_{\mathrm{c}}$ decreased significantly during the experiments from $2,300 \mathrm{~m} / \mathrm{s}$ at the free flyer edge to $600 \mathrm{~m} / \mathrm{s}$ after $4 \mathrm{~mm}$. Constantly changing collision conditions are well known phenomena in MPW and were proved and quantified with these experiments. Furthermore, the experiments showed that a weak flash occurred within the first millimeter after the initial impact, but was significantly intensified until $1.5 \mathrm{~mm}$. These findings correlate well with the unwelded zones reaching from 0 to $1 \mathrm{~mm}$ and from $3 \mathrm{~mm}$ to the end of the contact area, which are probably due to an insufficient flash intensity and value of $v_{\mathrm{c}}$, respectively.

It can be concluded that the presented method is an easily applicable and effective tool for process development in MPW. For the given joining geometry an increment of one millimeter was chosen to get a first impression of $v_{\mathrm{c}}$ and its development. Refining the graduation or repeating the experiments several times are two possibilities to increase the accuracy of the method. Even at this stage of development, it features a high information density with comparatively low preparation effort. The effect of changes in the joining setup, e.g. flyer thickness, acceleration gap, energy input, material properties, position in the working coil, or field shaper geometry, can be studied effectively in detail. Changing the parent's diameter gradually also allows for the determination of the impact velocity. Thus, the most relevant process parameters for MPW can be investigated and manipulated during process optimization with the presented measurement system.

\section{Acknowledgments}

This work is based on the results of subproject A1 of the priority program 1640 ("joining by plastic deformation"); the authors would like to thank the German Research Foundation (DFG) for its financial support.

\section{References}

[1] Carpenter, S., and Wittmann, R. 1975. "Explosive Welding." Annual Reviews of Materials Science 5: 177-99.

[2] Kapil, A., and Sharma, A. 2015. "Magnetic Pulse Welding: An efficient and Environmentally Friendly Multi-material Joining Technique." Journal of Cleaner Production 100: 35-58. doi: 10.1016/j.jclepro.2015.03.042.

[3] Lorenz, A., Lueg-Althoff, J., Bellmann, J., Göbel, G., Gies, S., Weddeling, C., Beyer, E., and Tekkaya, A. E. 2016. "Workpiece Positioning during Magnetic Pulse Welding of Aluminum-Steel Joints." Welding Journal [online] $\quad 95 \quad$ (3): $101-9$. https://s3.amazonaws.com/WJ-www.aws.org/supplement/ WJ_2016_03_s101.pdf. 
[4] Cuq-Lelandais, J.-P., Ferreira, S., Avrillaud, G., Mazars, G., and Rauffet, B. 2014. "Magnetic Pulse Welding: Welding Windows and High Velocity Impact Simulations." ICHSF2014, 199-206.

[5] Bellmann, J., Lueg-Althoff, J., Schulze, S., Gies, S., Beyer, E., and Tekkaya, A. E. 2016. "Measurement and Analysis Technologies for Magnetic Pulse Welding: Established Methods and New Strategies." Advances in Manufacturing 4: 322-339. doi: 10.1007/s40436-016-0162-5.

[6] Livshiz, Y., Gafri, O., and Izhar, A. 2002. "Magnetic Pulse Accelerators in Industry: Simulation and Practice." Pulsed Power Plasma Science, 1197-200.

[7] Poynton, W. A., Travis, F. W., and Johnson, W. 1968. "The Free Radial Expansion of Thin Cylindrical Brass Tubes Using Explosive Gas Mixtures.” International Journal of Mechanical Science 10: 385-401.

[8] Gafri, O., Izhar, A., Livshitz, Y., and Shribman, V. 2006. "Magnetic Pulse Acceleration." ICHSF2006, 33-40.

[9] Jäger, A., and Tekkaya, A. E. 2012. "Online Measurement of the Radial Workpiece Displacement in Electromagnetic Forming Subsequent to Hot Aluminum Extrusion." ICHSF 2012, 13-22.

[10] Lueg-Althoff, J., Schilling, B., Bellmann, J., Gies, S., Schulze, S., Tekkaya, A. E., and Beyer, E. 2016. "Influence of the Wall Thicknesses on the Joint Quality during Magnetic Pulse Welding in Tube-to-Tube Configuration." 7th International Conference on High Speed Forming - 2016, 259-68.

[11] Auluck, S. K. H., Kaushik, T. C., Kulkarni, L. V., and Rav, A. S. 2003. "Conical Electric Gun: A New Hypervelocity Macroparticle Launcher Based on the Munroe Effect." IEEE Transactions on Plasma Science 31 (4): 725-8.
[12] Bergmann, O. R. 1984. "The Scientific Basis of Metal Bonding with Explosives." "High Energy Rate Fabrication-1984" The 8th Int. ASME Conference, 197-202.

[13] Bellmann, J., Lueg-Althoff, J., Schulze, S., Beyer, E., and Tekkaya, A. E. 2016. "Magnetic Pulse Welding: Solutions for Process Monitoring within Pulsed Magnetic Fields." EAPPC \& BEAMS \& MEGAGAUSS 2016 Proceedings.

[14] Appelgren, P., Bjarnholt, G., Brenning, N., Elfsberg, M., Hurtig, T., Larsson, A., Novac, B. M., and Nyholm, S. E. 2008. "Small Helical Magnetic Flux-Compression Generators: Experiments and Analysis." IEEE Transactions on Plasma Science 36 (5): 2673-83. doi: 10.1109/TPS.2008.2003966.

[15] Seeberger. "Datasheet AlMgSi (EN AW-6060)." http://www.seeberger.net/_assets/pdf/werkstoffe/aluminiu $\mathrm{m} / \mathrm{de} / \mathrm{AlMgSi}$.pdf [retrieved 8 September 2016].

[16] Günther + Schramm. "Datasheet C45 (1.0503)." http://www.guenther-schramm-stahl.de/files/datasheets/C 45(1.0503).pdf [retrieved 8 September 2016].

[17] Bellmann, J., Lueg-Althoff, J., Göbel, G., Gies, S., Beyer, E., and Tekkaya, A. E. 2016. "Effects of Surface Coatings on the Joint Formation During Magnetic Pulse Welding in Tube-to-Cylinder Configuration." ICHSF 2016, 279-88.

[18] L'Eplattenier, P., Cook, G., and Ashcraft, C. 2008. "Introduction of an Electromagnetism Module in LS-DYNA for Coupled Mechanical Thermal Electromagnetics Simulations." ICHSF 2008, 85-96.

[19] Groche, P., Becker, M., and Pabst, C. 2017. "Process Window Acquisition for Impact Welding Processes." Materials \& Design 118: 286-93. 